\title{
Classification of Analyzable Metaphase Images by Extreme Learning Machines
}

\author{
Abdulkadir Albayrak \\ 1*Dicle University, Department of Computer Engineering, 21280, Sur, Diyarbakır, Turkey. (e-mail: kadir.albayrak@dicle.edu.tr).
}

\section{ARTICLE INFO}

Received: Oct., 29. 2020

Revised: Dec., 21. 2020

Accepted: March, 10. 2021

Keywords:

Karyotyping

Metaphase detection

Extreme learning machines

Gray level co-occurrence matrix

Corresponding author: Abdulkadir

Albayrak

ISSN: 2536-5010 | e-ISSN: 2536-5134

\section{ABSTRACT}

A chromosome is a DNA molecule that contains the genetic material of an organism. Possible defects in chromosomes can cause structural and functional disorders in living things. Identifying the metaphase stages of cells is a critical step to identify problems in chromosomes. In this proposed study, the discriminative features of possible metaphase images were extracted with Gray level co-occurrence matrix and classified with the Extreme Learning Machines classification method. When the results were evaluated, it was observed that the proposed method was as successful as the deep learning methods in the literature. Especially in recent years, when online learning has become important, the need for retraining of deep learning-based algorithms after each validation will increase the importance of the proposed method in this field. The rapid increase in unlabelled data from each patient every day affects the duration of training and creates time and resource constraints. Fast and accurate modelling of such data with alternative machine learning methods will contribute to the studies in this area.

DOI: https://doi.org/10.36222/ejt.818160

\section{INTRODUCTION}

Cytogenetics is a branch of genetics which concerned with the study of number, structure and function of chromosomes. A healthy individual has a total of 46 chromosomes, 23 of which are genetically inherited from the mother and 23 from the father. Anomalies in these chromosomes may cause the presence of various syndromes called congenital (Down syndrome, Edward syndrome, etc.). Experts try to detect the anomaly by analyzing genes obtained by sampling special tissues such as bone marrow, blood, amniotic fluid or placenta in order to detect possible congenital syndromes. This process performed in the expert's cytogenetics laboratory is called karyotyping. In karyotyping, chromosome analyzes are performed based on the division of cells in the metaphase stages of the samples taken from the patient. Scanning of chromosomes on the specimen slide is done at the metaphase stage, since the chromosomes have the best morphological features while in the metaphase stage and are suitable for imaging. At least 8 to 10 glass slide samples should be prepared for each individual in order to obtain an adequate analyzable metaphase spread image. Each glass slide typically contains about 10-20 metaphase formations. Of the approximately 200 metaphases prepared, approximately 20 of the "best" (based on the subjective opinion of an experienced cytogenetics expert) are selected for karyotyping. Optical microscopes are used for image selection and analysis. The lens of the microscope are the apparatus that determines the quality of the image obtained, since it controls the illumination and the angle of entry of the light into the front lens. It has been observed that by adjusting the aperture of the lenses, more analyzable chromosome images can be obtained. The karyotyping process, which is quite tiring for the expert, also affects the performance of the expert in the process, and misdiagnosis may be made due to fatigue and lack of performance. In recent years, especially with the development of imaging technology and digital image processing methods, it is aimed to develop secondary decision support systems that will help the expert by making the karyotyping process, which is difficult and time-consuming by the expert with the help of computer-aided diagnosis systems. There are various studies proposed in the literature to handle this purpose. These approaches can generally be divided into two groups as rulebased and deep learning-based image processing methods [1]. When the studies in the literature are examined in rule-based methods, it can be observed that the rates of false positive are quite high [2]. In recent years, It has been observed that the deep learning methods give accurate results in many areas such as object recognition, target tracking, natural language processing, voice recognition [3].

The number of images used in previous studies are presented comparatively in Table 1 [3-16]. Difficulties in preparing data and the high false positive rates of the achieved achievements prevented the creation of a data set that can be considered as a reference in this area. The number of images 
used in this area is expected to increase due to the following reasons: Especially in recent years, devices with high imaging technology used for this purpose in hospitals' genetic laboratories and existence of high-performance devices, especially using up-to-date digital image processing methods. After deep learning-based metaphase detection methods such as GoogleNet, VGG16, Inception, LeNet, which were quite successful in our previous study in the literature and we suggested before, experts were provided to present metaphase images with higher success.

TABLE I

THE NUMBER OF IMAGES USED IN THE LITERATURE AND IN THIS PROPOSED METHOD

\begin{tabular}{ll}
\hline \hline Proposed study & \# of images \\
\hline Castleman 1992 [4] & - \\
Garza Jinich et. al. 1992 [5] & 1,196 \\
Vroluk et. al. 1994 [6] & 150 \\
Mclean and Johnson1995 [7] & 100 \\
Corkidi et. al. 1998 [8] & 177 \\
Cosio A et. al. 2001 [9] & 909 \\
Wang et. al. 2008 [10] & 170 \\
Ravi Uttamatanin 2013 [11] & 200 \\
Yucehn Q et. al. 2014 [12] & 200 \\
Yucehn Q et. al. 2016 [13] & 150 \\
Tanvi Arora, RenuD 2017 [14] & 200 \\
Y1lmaz and Turan 2017 [15] & 3018 \\
Y1lmaz and Turan 2018 [16] & 3018 \\
Moazen et. al. [3] & 50,000 \\
Proposed & 50,000 \\
\hline \hline
\end{tabular}

\section{MATERIALS AND METHODS}

This proposed study consists of two stages: Detection of metaphase candidates and classification of metaphase candidates in order to identify possible metaphases.

\subsection{Detection of Metaphase Candidates}

The imaging system used in the study consists of the following components: Zeiss Imager M2 motorized microscope with 0.3 numerical aperture, $10 x$ objective and $0.63 x$ trinocular camera adapter. 1.4 megapixel (1360 x 1024) CCD camera with Marzhauser scan and 2/3 inch for $8 \mathrm{X}$ - Y motorized stages. The first stage, which determines the possible metaphases in this proposed study, consists of 5 basic sub-steps.

In the first step, the system performs noise removal pretreatment using a low pass filter to reduce the noise ratio of the scanned image. In the second step, the gradient amplitude of the pixels is found by the Sobel method in order to detect the edges of the cells and objects in the image. In the third step, the Otsu method is used to determine and reduce the color levels of the gray levels [17]. In the fourth step, irrelevant objects such as spots and undivided cells are eliminated from the image. The system performs this step by using the morphological properties of these objects such as their circularity and pixel information. Finally, using the morphological dilation process, neighboring chromosomes of the metaphase cells are linked and the cell regions (region of interest) that have fully metaphase spread are recorded to the computer. In order to verify the steps performed at this stage, it scans the 100x zoomed image of the regions of interest obtained and provides performance improvement by eliminating the regions that are thought to have background information. Figure. 1 obtains a whole image obtained from the data set and the metaphase and non-metaphase image sections obtained from this image. Figure 1 represents the first step of the proposed method, which is the detection of metaphase candidates.

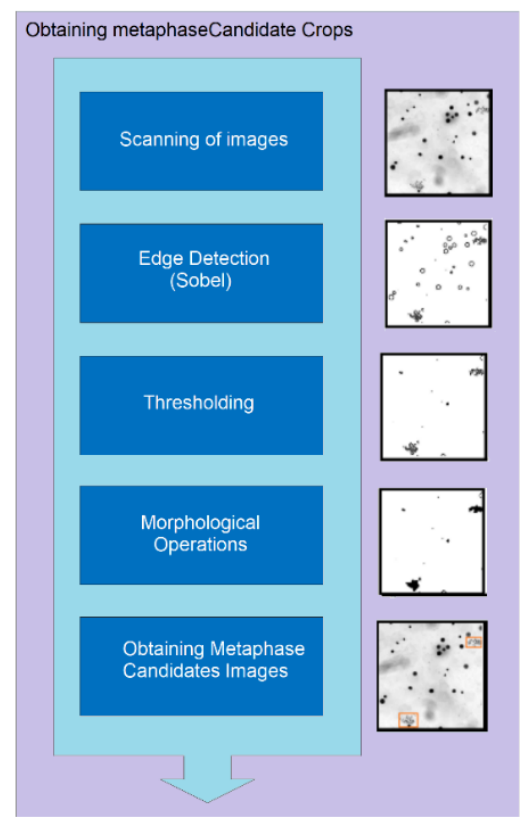

Figure 1. Basic operation steps of the first stage of the proposed method.

\subsection{Classification of Metaphase Candidates}

After obtaining the possible metaphase sections highlighted in the previous section, it is necessary to obtain distinctive features in order to perform high-performance classification. Traditional methods are used to obtain shape, color and textural features in the image that is desired to be distinguished in the region of interest. Depending on the characteristics of the object to be classified, features such as the longest side, the shortest side, the ratio of these edges to each other, concave and convexity define the shape information; the red, green and blue pixel brightness values describe the color information. In some images where shapes and colors are not distinctive, tissue information (such as a change in the pattern of a cancerous region due to cell density) is tried to be obtained with textural information descriptors. These highlighted methods are defined specific to the problem and implemented manually to solve the current problem.

\subsubsection{Gray Level Co-Occurrence Matrix(GLCM)}

Feature vectors of possible metaphase image sections included in the data set were extracted using the gray level co-occurrence matrix (GLCM). GLCM is a texture descriptor algorithm proposed by Robert Haralick [18]. The algorithm basically tries to obtain a distinctive feature between images by calculating the number of sequences of certain successive pixels. This method takes a gray level image as input. In an input image, the values of consecutive pixels are computed and thus the GLCM features are extracted. Then, specific vectors are taken from this GLCM and given as input to the classification method. In order to obtain features by using GSEM in color images, the features can be obtained by applying the algorithm to each color band separately. One of the important parameters used in the algorithm is at what angles the method will be applied. For example, if it is desired to be applied at 45 degrees in the whole image, the number of the central pixel and the other pixels adjacent to that pixel at 


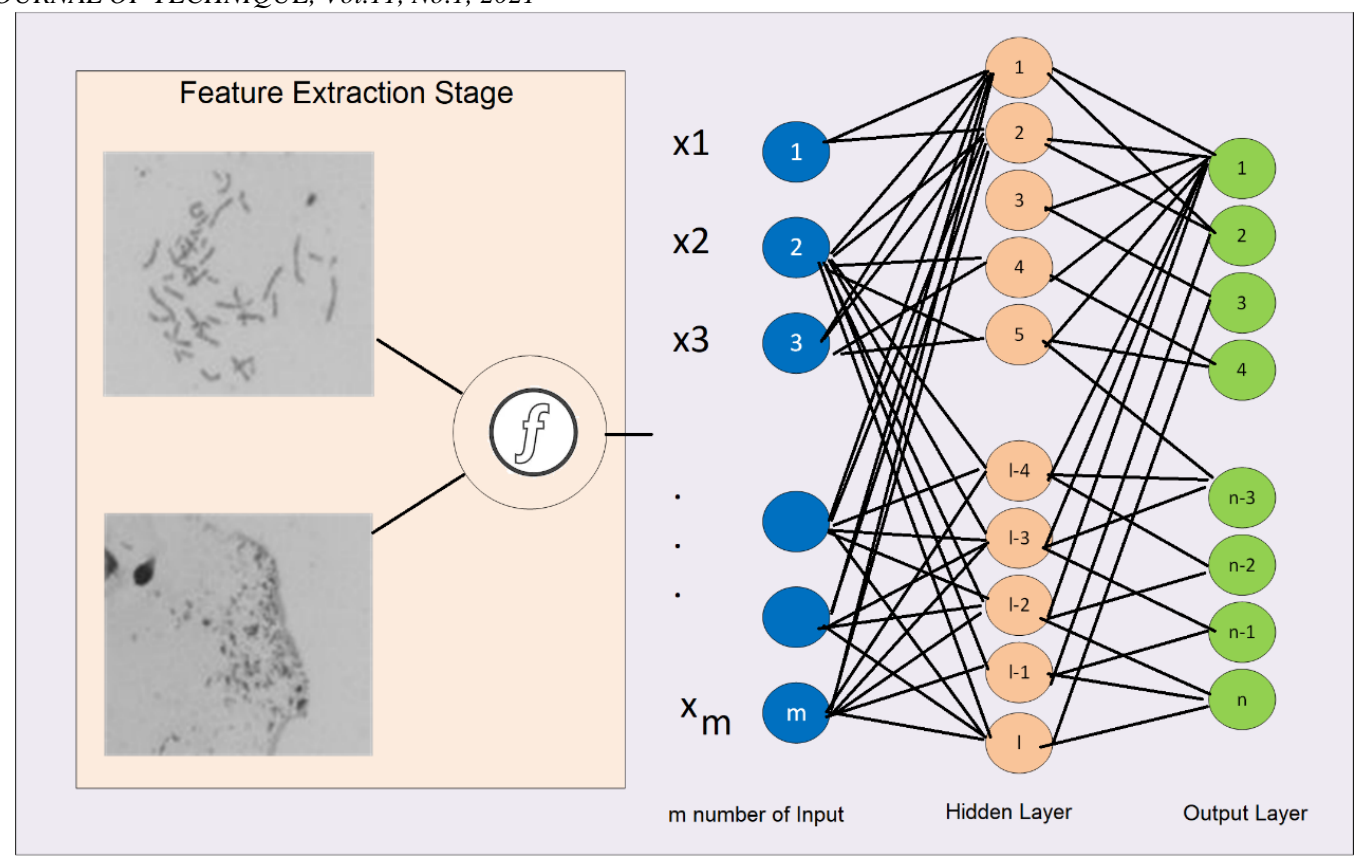

Figure 2. Classification stage of the proposed study

45 degrees are kept in the formation matrix. After applying Figure 2: Classification stage of the proposed study this process to all pixels in the image, the resulting co-occurrence matrix is then used for feature extraction. In this study, GLCM. was calculated by counting the sequences of pixels in 4 neighborhoods in one direction and 10 feature vectors related to each image were obtained.

\subsubsection{Extreme Learning Machines (ELM)}

Extreme Learning Machine (ELM) is a machine learning method that consists of a single feed-forward neural network and classifies objects based on their distinctive properties. [19]. ELM was first designed to consist of a total of 3 layers, as an input layer, a hidden layer and an output layer. Unlike convolutional neural networks, weights connecting hidden nodes to outputs can be trained very quickly. It can be said that it has only one hidden layer and the fast results are obtained with vector products. When the experimental studies in the literature are examined, it has been shown that ELMs can produce acceptable predictive performance and the computation costs are much lower than the networks trained by the back propagation algorithm. Figure 2 shows the process steps followed in metaphase classification in this proposed study.

$$
X=x_{t} \in R^{p} t=1, \ldots, n
$$

In Eq. 1, let $\mathrm{R}^{\wedge} \mathrm{p}$ be a dataset to which ELM will be applied, with $\mathrm{n}$ number of samples and $\mathrm{p}$ features. The outputs to be obtained with ELM can be expressed in Eq. 2.

$$
O_{i}\left(x_{t}\right)=m_{i}\left(x_{t}\right) h\left(x_{t}\right)
$$

where $\mathrm{m} \_\mathrm{i}$ indicates the weight vector connecting the latent neurons to the $\mathrm{i}$ th output neuron. $\mathrm{h}(\mathrm{t})$ represents the vector of the outputs of hidden neurons for a given input model x_t. Then $\mathrm{h}(\mathrm{t})$ can be written as:

$$
\begin{aligned}
h(t)=f\left(w_{1}^{T} x(t)\right. & \left.+b_{1}\right), f\left(w_{2}^{T} x(t)\right. \\
& \left.+b_{2}\right), \ldots, f\left(w_{q}^{T} x(t)+b_{k}\right)
\end{aligned}
$$

Here, $b \_k$ indicates the bias of the k'th latent neuron, $w \_k$ represents the weight vector of the k'th latent neuron and $\mathrm{f}($.). indicates a sigmoidal activation function. An important point to note here is that the values of the weight vectors $\mathrm{w}_{-} \mathrm{k}$ and the bias $b \_k$ are randomly generated from a Gaussian distribution.

\section{EXPERIMENTS}

The digital images used in this proposed study were first published by Moazzen et. al. [3]. There are 55000 possible metaphase image sections obtained from a total of 26000 images with a width and height of $1360 \times 1024$ to create the data set. The location of possible metaphases in these images has been precisely marked by a sitogenetic expert.

In Table 2, the comparison of true positive rate (TPR) and false positive rate (FPR) values of previous studies were compared with the proposed study. According to the table, while the FPR in the results obtained with traditional methods such as SVM, decision trees and k-NN is quite high, the FPR of deep learning methods remained quite low. In addition, the TPR in the table have yielded more successful results in deep learning approaches. It has been observed that the proposed method gives more successful results than traditional methods, but relatively less successful than deep learning-based methods. Considering the FPR values, it can be said that the proposed method has a low error rate. In the proposed method, the training and classification phase is completed in 239 seconds in total, while in our previous study [3], where the data set was shared, it can take hours for deep learning methods. Considering the rapid increase in data, the need to train the system over and over again for each new data requires the development of alternative methods to deep learning algorithms. 


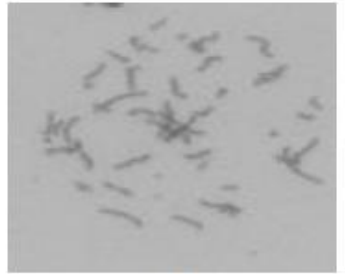

(a)

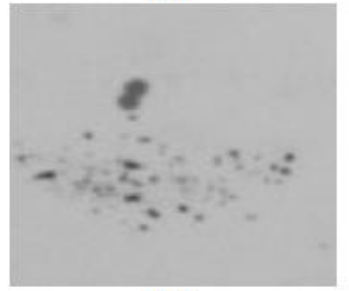

(c)

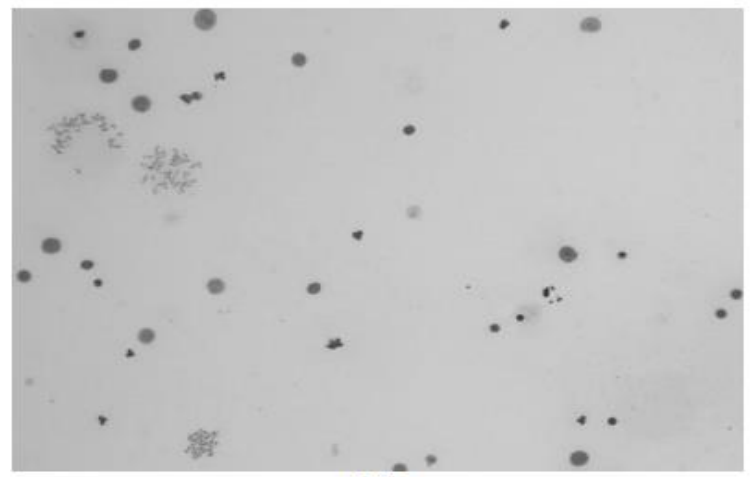

(e)

Figure 3. (a) and (b) represent metaphase images obtained from dataset; while (c) and (d) represent non-metphases images. (e) represents whole image which includes metaphases and non-metaphase image crops.

Table 2. Comparison of TPR and FPR values of the proposed method with the methods in the literature

TABLE 2

THE NUMBER OF IMAGES USED IN THE LITERATURE AND IN THIS PROPOSED METHOD

\begin{tabular}{lcl}
\hline \hline Approaches & TPR & FPR \\
\hline SVM based & $\% 88$ & $\% 69$ \\
Decision Trees & $\% 89$ & $\% 71$ \\
k-NN based & $\% 90$ & $\% 72$ \\
AlexNet & $\% 99$ & $\% 0.76$ \\
GoogleNet & $\% 98$ & $\% 0.52$ \\
ResNet & $\% 99$ & $\% 0.74$ \\
Inception & $\% 99$ & $\% 0.55$ \\
Castlemen & $\% 80$ & $\% 0.73$ \\
MetaSel & $\% 90$ & $\% 4.6$ \\
proposed & $\% 97,6$ & $\% 2.2$ \\
\hline \hline
\end{tabular}

It is a known fact both in this study and in the literature that deep learning methods are more successful than traditional methods. However, it is a known fact that the training phase of deep learning algorithms in large-scale data is very slow if the graphics processing unit is not used. With the graphic processing unit, the training phase is very fast, but the repetitive training of the data obtained from patients, such as the data set used in this study, and increasing day by day, can make deep learning algorithms inefficient in terms of time. It is an undeniable fact that the training phase gain importance as the amount of data increases.

\section{CONCLUSION}

In this proposed study, the feature vectors of the possible metaphase images were extracted with the GLCM algorithm and classified with the ELM algorithm. When the results were evaluated, it was observed that performance of the proposed method has more accurate than results obtained with rule-based algorithms. In our first study where the dataset was introduced, a very high success was achieved with deep learning-based algorithms. It is clear that algorithms based on deep learning have the best results in classifying metaphase images. However, the long duration of the training phase of deep learning algorithms and the need for retraining, especially after the verification of data such as online learning, caused the need to examine alternative methods. ELM differs from deep learning algorithms in this respect. With the GLCM and single layer Basic-ELM algorithm, the training and classification phase of the data takes in 239 seconds in total. It is clear that this approach, which is quite fast in this respect, will be a good alternative by increasing the classification performance. In future studies, testing algorithms such as kernel-based ELM (Kernel ELM), multilayer ELM (Multilayer ELM), which can be alternative to Basic-ELM in classification of metaphase images, with different feature extraction algorithms may increase the performance. In addition, the training processes will be accelerated by examining transfer learning and fine tuning processes. It is planned to focus on these methods in future studies.

\section{ACKNOWLEDGEMENT}

The Author would like to thank Argenit Co. for their contribution in providing and marking the data.

\section{REFERENCES}

[1] X. Wang, B. Zheng, M. Wood, S. Li, W. Chen, \& H. Liu, "Development and evaluation of automated systems for detection and classification of banded chromosomes: current status and future perspectives," Journal of Physics D: Applied Physics, 38(15), 2536, 2005.

[2] T. Arora, \& R. Dhir, "A review of metaphase chromosome image selection techniques for automatic karyotype generation," Medical \& Biological Engineering \& Computing, 54(8), 1147-1157, 2016.

[3] Y. Moazzen, A. Çapar, A. Albayrak, N. Çalık, \& B. U. Töreyin, "Metaphase finding with deep convolutional neural networks," Biomedical Signal Processing and Control, 52, 353-361, 2019.

[4] K. R. Castleman, "The PSI automatic metaphase finder," Journal of radiation research, 33(Suppl_1), 124-128, 1992.

[5] M. Garza-Jinich, C. Rodriguez, G. Corkidi, R. Montero, E. Rojas, \& P. Ostrosky-Wegman, "A Microcomputer-Based Supervised System for Automatic Scoring of Mitotic Index in Cytotoxicity Studies," In Advances in Machine Vision: Strategies and Applications (pp. 301311), 1992.

[6] J. Vrolijk, W. C. R. Sloos, F. Darroudi, A. T. Natarajan, \& H. J. Tanke, "A system for fluorescence metaphase finding and scoring of chromosomal translocations visualized by in situ hybridization," International journal of radiation biology, 66(3), 287-295, 1994.

[7] J. R. N. McLean \& F. Johnson, "Evaluation of a metaphase chromosome finder: Potential application to chromosome-based radiation dosimetry," Micron, 26(6), 489-492, 1995.

[8] G. Corkidi, L. Vega, J. Márquez, E. Rojas, \& P. Ostrosky-Wegman, "Roughness feature of metaphase chromosome spreads and nuclei for automated cell proliferation analysis," Medical \& Biological Engineering \& Computing, 36(6), 679-685, 1998.

[9] F. A. Cosío, L. Vega, A. H. Becerra, R. P. Meléndez, \& G. Corkidi, "Automatic identification of metaphase spreads and nuclei using neural networks," Medical \& Biological Engineering \& Computing, 39(3), 391-396, 2001. 
[10] X. Wang, S. Li, H. Liu, M. Wood, W. R. Chen, \& B. Zheng, "Automated identification of analyzable metaphase chromosomes depicted on microscopic digital images" Journal of Biomedical Informatics, 41(2), 264-271, 2008.

[11] R. Uttamatanin, P. Yuvapoositanon, A. Intarapanich, S. Kaewkamnerd, R. Phuksaritanon, A. Assawamakin, \& S. Tongsima, "MetaSel: a metaphase selection tool using a Gaussian-based classification technique," BMC bioinformatics, 14(16), 1-13, 2013

[12] Y. Qiu, J. Song, X. Lu, Y. Li, B. Zheng, S. Li, \& H. Liu, "Feature selection for the automated detection of metaphase chromosomes: performance comparison using a receiver operating characteristic method," Analytical Cellular Pathology, 2014.

[13] Y. Qiu, X. Lu, S. Yan, M. Tan, S. Cheng, S. Li, ... \& B. Zheng, "Applying deep learning technology to automatically identify metaphase chromosomes using scanning microscopic images: an initial investigation," International Society for Optics and Photonics(Vol. 9709, p. 97090K), 2016

[14] Arora, T., \& Dhir, R. (2017). An automatic human chromosome metaspread image selection technique. Knowledge and Information Systems, 52(3), 773-790.

[15] H. Yilmaz, \& M. K. Turan, "FahamecV1: A Low Cost Automated Metaphase Detection System," Engineering, Technology \& Applied Science Research, 7(6), 2160-2166, 2017.

[16] H. Yilmaz, \& M. K. Turan, "Filter development for automatic detection of analyzable metaphases," In 2018 26th Signal Processing and Communications Applications Conference (SIU) (pp. 1-4), 2018.

[17] X. Xu, S. Xu, L. Jin, \& E. Song, "Characteristic analysis of Otsu threshold and its applications," Pattern recognition letters, 32(7), 956961, 2011.

[18] R. M. Haralick, \& L. G. Shapiro, "Computer and robot vision" Reading: Addison-wesley, (Vol. 1, pp. 28-48), 1992.

[19] G. B. Huang, Q. Y. Zhu, \& C. K. Siew, "Extreme learning machine: theory and applications," Neurocomputing, 70(1-3), 489-501, 2006

\section{BIOGRAPHIES}

Abdulkadir Albayrak completed BSc, MS and $\mathrm{PhD}$ in computer science. $\mathrm{He}$ is a Research Assistant at the Department of Computer Engineering, Dicle University, Turkey. He studies machine learning, computer vision and biomedical image analysis. 